\title{
The value of exosomes in patients with laryngeal or hypopharyngeal cancer
}

\author{
Yuan-Jing Jia ${ }^{\mathrm{a}}$, Hui-Hua You ${ }^{\mathrm{a}, *}$, Shui-Hong Zhou ${ }^{\mathrm{b}}$ \\ a Department of Otolaryngology, Zhejiang University Jinhua Hospital, Jinhua 321000 China \\ b Department of Otolaryngology, First Affiliated Hospital of Zhejiang University School of Medicine, \\ Hangzhou 310009 China
}

*Corresponding author, e-mail: youhh692174@163.com

Received 28 Feb 2020

Accepted 3 Dec 2020

\begin{abstract}
This study aimed to evaluate the relevance of exosomes in laryngeal or hypopharyngeal cancer. The peripheral blood from 22 patients with head or neck cancer and 11 normal subjects were retrospectively analyzed. The exosomes were extracted and the shape was observed through an electronic transmission microscope. Nanoparticle Tracking Analysis (NTA) was used to detect the particle concentration and particle size of the exosomes. The exosomes from the supernatant liquids of laryngeal cancer and hypopharyngeal cancer patients could be separated. They had a cup-shaped structure with the diameters within 30-100 nm. The protein concentration of exosomes from both types of cancer patients was significantly higher than from normal subjects $(p<0.05)$. Using western blot, Cluster of differentiation (CD) 63 and CD 81 were detected in the exosomes, but protein calnexin was not detected. The particle concentration of exosomes of the patients was not significantly different from normal subjects $(p>0.05)$. The particle diameter of exosomes from laryngeal cancer patients was larger than that from normal subjects $(p>0.05)$ while that from hypopharyngeal cancer patients was significantly larger $(p<0.01)$. In conclusion, exosomes were expected to be a potential non-invasive and simple tumor marker of body fluid for laryngeal or hypopharyngeal cancer.
\end{abstract}

KEYWORDS: Laryngeal cancer, hypopharyngeal cancer, exosomes, tumor marker, malignant tumor

\section{INTRODUCTION}

Laryngeal malignant tumor is one of the most common malignant tumors in humans [1]. Generally, laryngeal malignant tumor includes laryngeal and hypopharyngeal cancers [2]. The pathology is mostly squamous cell cancer. The symptoms of laryngeal malignant tumors in the early phrase are not typical, and these can easily lead to cervical lymph node metastasis, and a poor prognosis. Therefore, the early detection, early diagnosis and early treatment of laryngeal and hypopharyngeal cancers are extremely important for such patients, in order to improve their survival rate and life quality [3-5]. Seeking for tumor markers is the key for the diagnosis, treatment and prognosis of such tumors in the early phase $[6,7]$.

Exosomes, 30-150 nm mature double membrane multivesicular bodies (MVBs) originating from the endosomal pathway, are highly expressed in blood and body fluids such as urine and saliva [8]. Growing evidence has demonstrated that exosomes participate in the development, progression and treatment of cancer by mediating intercellular communication and signal transduction [9]. Thus, exosomes may serve as promising markers for monitoring cancer progression and therapeutic responses $[10,11]$. Furthermore, exosomes can be obtained from blood or saliva of patients, thus, the detection method is relatively simple and noninvasive $[12,13]$. In head and neck cancers, previous studies have demonstrated that the number of exosomes in the body fluids could be used as a prognosis indicator. Gimzewski et al reported that elevated exosome numbers, exosome sizes, and interexosomes were detected in the saliva of patients with oral cancer; and patients with advanced-stage disease and shorter overall survival exhibited elevated levels of exosomes [14]. Kannan et al demonstrated that exosome signatures could serve as candidate biomarkers for early cancer diagnosis, monitoring and surveillance in HPV-16-associated oropharyngeal [15]. But, currently, the role of exosomes in laryngeal or hypopharyngeal cancer has not been determined. Therefore, this study aims to evaluate the relevance of exosomes in laryngeal or hypopharyngeal cancer. 


\section{MATERIALS AND METHODS}

\section{General consideration}

This was a retrospective study to evaluate the relevance of exosomes in laryngeal or hypopharyngeal cancer.

\section{Compliance with ethics guidelines}

This study was conducted in accordance with the Declaration of Helsinki and approved by the Ethics Committee of Zhejiang University Jinhua Hospital. Since the study does not require any patient-related intervention or experiment, the Ethics Committee has reviewed it without informed consent.

\section{Population}

From 2015 to 2017, patients with laryngeal malignant tumors who were treated in the Department of Otolaryngology of Jinhua Hospital had been recruited in this study. Approval by the Ethics Committee of the hospital was performed; and all patients provided a signed informed consent.

\section{Inclusion and exclusion criteria}

Inclusion criteria: (1) patients who were diagnosed as laryngeal or hypopharyngeal cancer; (2) patients with primary cancer; (3) patients $>18$ years old. Exclusion criteria: (1) patients who received radiotherapy or chemotherapy before the surgery; (2) patients whose tumors have already metastasized.

\section{Extraction and characterization of exosomes}

The experimental groups: (1) the peripheral blood serum of normal healthy people was used as the control group; (2) the peripheral blood serum from laryngeal cancer patients was taken as the laryngeal cancer group; (3) the peripheral blood serum from hypopharyngeal cancer patients was taken as the hypopharyngeal cancer group.

An Exosome Extraction Kit (EXOTC10A-1, System Biosciences, CA, USA) was used to extract and culture the serum. The first stage: the serum was centrifuged at $3000 \mathrm{~g}$ for $15 \mathrm{~min}$, and the supernatant is collected. The second stage: Add $450 \mu \mathrm{l}$ of precipitant $(4: 1)$ to the liquid obtained in the first step. After even mixing, it was fixed for $30 \mathrm{~min}$ at $4{ }^{\circ} \mathrm{C}$. The third stage: the incubated samples are centrifuged at $1500 \mathrm{~g}$ for $5 \mathrm{~min}$, and the supernatant was discarded. The fourth stage: the precipitation was suspended by $100 \mu$ l of phosphate buffered saline (PBS), repackaged, and kept at $-80^{\circ} \mathrm{C}$.

The exosomes were observed through an electronic transmission microscope. Basic steps: Fifty $\mu 1$ of exosome suspension was added to red wax, a polyvinyl acetate/carbon coated copper net was then added; and this was fixed for $20 \mathrm{~min}$ at room temperature. The excess liquid was absorbed on filter paper and fixed with $2 \%$ paraformaldehyde ( $2 \%$ PFA) for $2 \mathrm{~min}$. The copper mesh was washed 3 times with double distilled water and re-stained with $2 \%$ phosphotungstic acid (2\% PTA) for $1 \mathrm{~min}$. The excess liquid was absorbed on filter paper and dried overnight at room temperature.

The BCA protein was quantified according to the serum sample size. The bicinchoninic acid (BCA) working fluid was prepared using (50:1) BCA reagents $A$ and $B$. The $B C A$ working fluid was stable at room temperature for $24 \mathrm{~h}$. The BSA standard was used at 1.0, 0.8, 0.6, 0.4, and $0.2 \mathrm{mg} / \mathrm{ml}$. Add $20 \mu \mathrm{l}$ of serum samples at various dilutions in PBS or standard protein samples at various concentrations to a 96-well plate. Afterwards, $200 \mu \mathrm{l}$ of BCA working liquid was added into each pore, and these are fixed for $30 \mathrm{~min}$ at $37^{\circ} \mathrm{C}$. The absorbance at $595 \mathrm{~nm}$ was measured.

Western blot detection: exosomes were lysed, denatured in SDS buffer (0.4\% SDS, 0.2 M Tris$\mathrm{HCl}, \mathrm{pH}$ 6.8, 5\% glycerol, $0.02 \%$ bromophenol blue, $1 \% 2$-mercaptoethanol) at $95^{\circ} \mathrm{C}$ for $10 \mathrm{~min}$. Equal amounts of extracted protein (50 $\mu \mathrm{g} /$ lane) were separated by $10 \%$ SDS-PAGE and transferred to polyvinylidene difluoride membranes (BioTrace NT, Darmstadt, Germany). The membranes were blocked for $2 \mathrm{~h}$ with $5 \%$ non-fat milk powder and, afterwards, incubated overnight at $4{ }^{\circ} \mathrm{C}$ with antibodies against CD63 (1:1000), CD81 (1:1000) and Calnexin $(1: 1000)$. A secondary antibody (horseradish peroxidase (HRP)-conjugated goat anti-mouse IgG) was applied for $1 \mathrm{~h}$ at ambient temperature. Luminescent visualization was done using an ECL kit (Merck Millipore, Darmstadt, Germany) to identify immunoreactive protein bands.

\section{Statistical methods}

The SPSS 19.0 software program was used to perform the statistical analysis. Continuous variables were expressed as mean \pm standard deviation (SD). Discontinuous variables were expressed in percentage (\%). For two comparisons, each value was compared by $t$-test when each datum conformed to the normal distribution; while non-normally distributed continuous data were compared using nonparametric tests. Counting data were analyzed by chi-square test. The receiver operating characteristic (ROC) curve analysis of the subject work characteristics was used to determine whether the head 


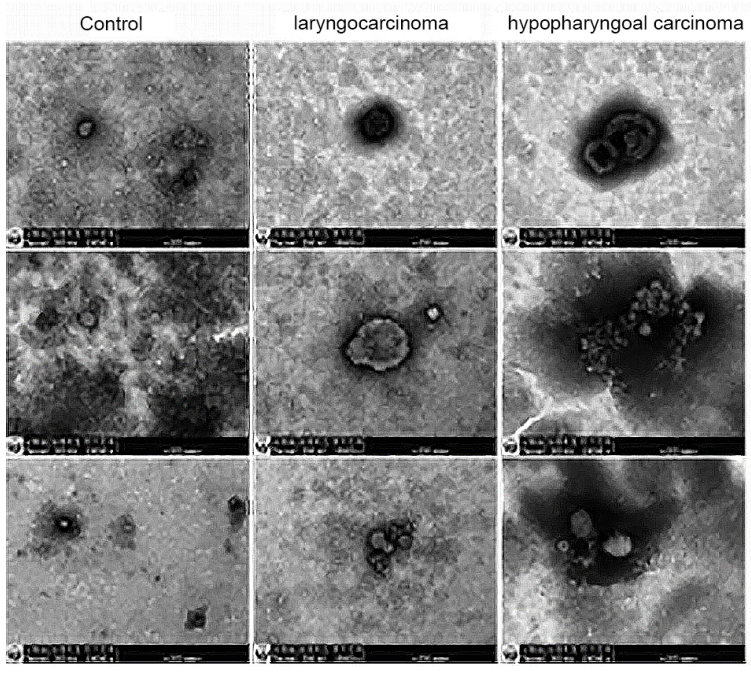

Fig. 1 The morphology and volume of exosomes were assessed by electronic transmission microscopy.

and neck lesions were benign or malignant. In order to calculate the area under the ROC curve and the corresponding Youden index, the following formula was used: sensitivity + specificity $-1 . p<0.05$ was considered statistically significant.

\section{RESULTS}

\section{General clinical data}

In the present study, a total of 22 patients with head and neck malignancies were included. All of these 22 patients were male, and their age ranged within 50-77 years old, with an average age of 64.5 years old. Among these patients, 11 cases had laryngeal cancer ( 9 cases were glottic site and 2 supraglottic site) and the other 11 cases had hypopharyngeal cancer ( 9 cases were piriform fossa type and 2 posterior pharyngeal wall type). In addition, 3 cases were severe heterogenous hyperplasia and focal cancer; the remaining 19 cases were squamous cell cancer. TNM stages: 6 cases were T1NOM0; T1N1M0, 1 case; T2NOM0, 2 cases; T2N1M0, 2 cases; T3NOM0, 1 case; T3N1M0, 4 cases; T3N2M0, 4 cases; T4NOM0, 1 case; and T4N2M0, 1 case. Clinical staging: 6 cases $(27.3 \%)$ were in stage I, 3 cases $(13.6 \%)$ in stage II, 3 cases (13.6\%) in stage III, and 10 cases $(45.5 \%)$ in stage IV.

\section{The exosomes were tested by electronic transmission microscopy}

The present study, the morphology of exosomes was verified by electronic transmission microscopy. The results revealed that the lipid double wrapped flat
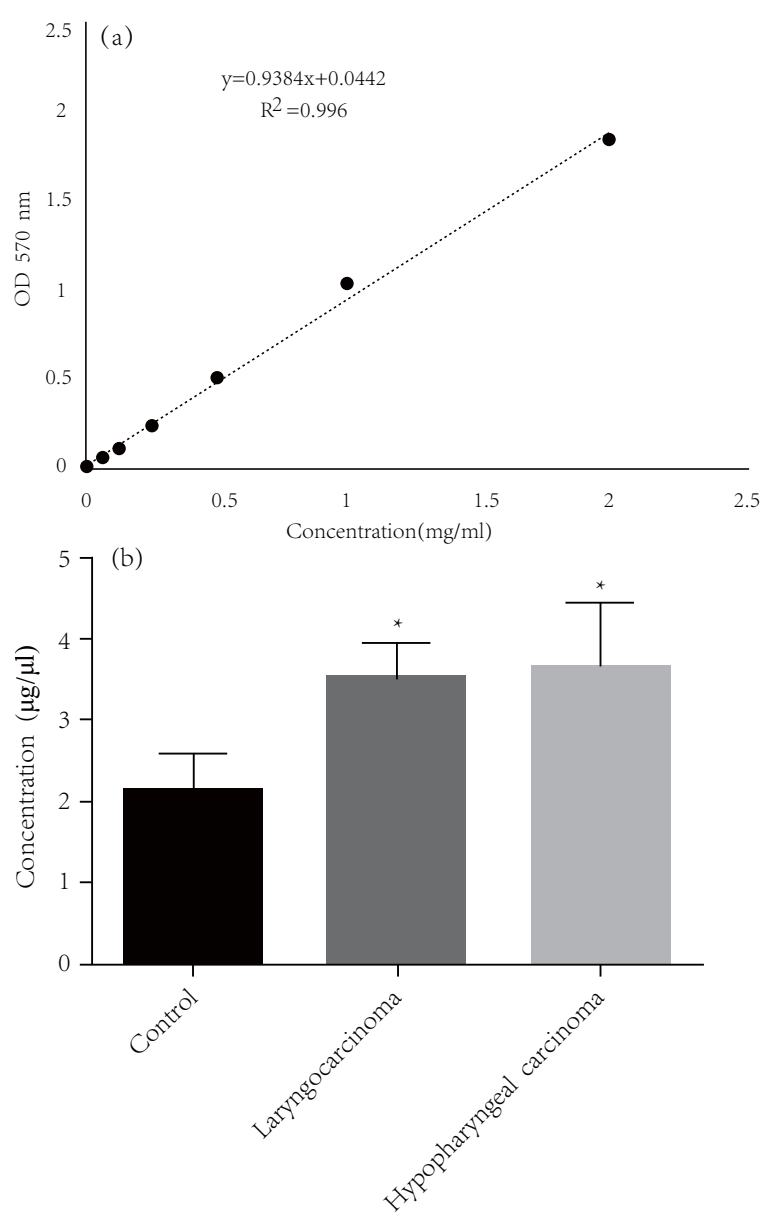

Fig. 2 Exosome protein concentration as measured by BCA (* $p<0.05$ versus control).

sphere, diameter of the $30-100 \mathrm{~nm}$, presented with a cup-shape structure, which was in line with the typical features of exosomes.

Fig. 1 shows that the exosome volume was larger in patients with laryngeal or hypopharyngeal cancer, when compared with the peripheral blood serum of normal subjects.

\section{Exosome protein concentration was measured by BCA protein}

Fig. 2 shows that the exosome protein concentration obviously increased in both the laryngeal and hypopharyngeal cancer groups, when compared with the control group $(p<0.05)$.

Area under the curve: AUC $=0.926 \pm 0.046$. When the exosome protein concentration was $2.8842 \mathrm{mg} / \mathrm{ml}$, the maximum Youden index was 0.864 , the diagnostic sensitivity was $86.4 \%$, and the specificity was $100 \%$. Therefore, an exosome pro- 


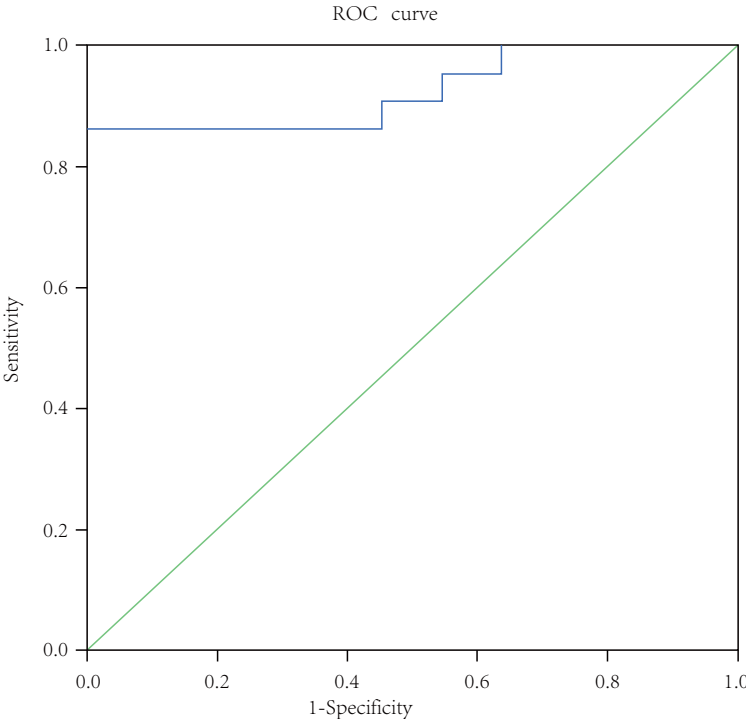

Fig. 3 ROC curve results [protein concentration] : control group and malignant tumor group [laryngeal cancer group + hypopharyngeal cancer group].

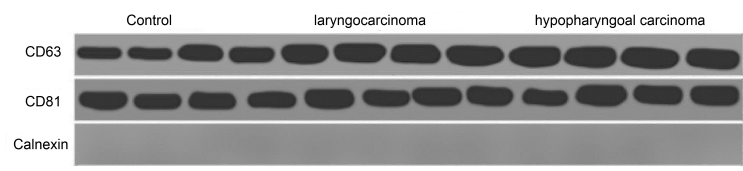

Fig. 4 Expression of CD63, CD81 and calnexin in exosomes as determined by Western blot. The results show 4 replicates (4 lanes) of each sample.

tein concentration of $2.8842 \mathrm{mg} / \mathrm{ml}$ was determined as the diagnostic threshold, and the exosome protein concentration in peripheral blood can be used as an indicator for the early diagnosis of laryngeal and hypopharyngeal cancers (Fig. 3).

The protein expression of CD63, CD81 and calnexin in exosomes was determined by western blot

Fig. 4 shows that CD63 and CD81 proteins were detected in exosomes from the control group, laryngeal cancer group and hypopharyngeal cancer group, while calnexin protein was not detected.

The exosome particle concentration and particle size were measured by NTA

As shown in Fig. 5, there was no significant difference in exosomes particle concentration in the control group, laryngeal cancer group and hypopharyngeal cancer group $(p>0.05)$. Compared to the control group, the exosome particle size of

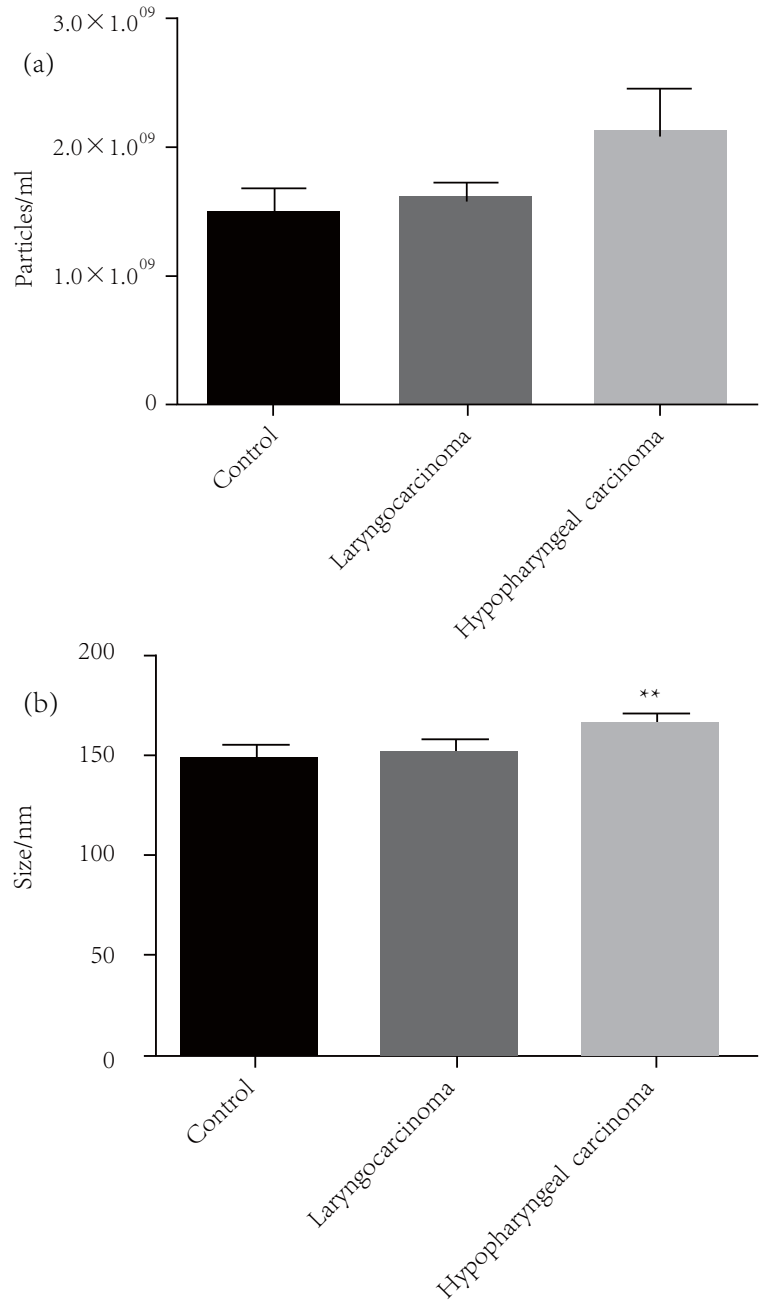

Fig. 5 The exosomes particle concentration and particle size within $50-250 \mathrm{~nm}(* * p<0.01$ versus control).

laryngeal cancer non-significantly increased $(p>$ 0.05 ). On the other hand, exosome particle size significantly increased in the hypopharyngeal cancer group, and the difference was statistically significant $(p<0.01)$. The ROC curve result for the exosome particle size in the control group and hypopharyngeal cancer group were analyzed as follows: AUC $=$ $0.979 \pm 0.024$. When the exosome particle size was $162 \mathrm{~nm}$, the largest Youden index was 0.818 . Therefore, the exosome particle size value of $162 \mathrm{~nm}$ was taken as the critical value, the diagnostic sensitivity was $100 \%$, and the specificity was $81.8 \%$. Hence, the exosome particle size in peripheral blood can be used as one of the indexes for the early diagnosis of hypopharyngeal cancer. 


\section{DISCUSSION}

Laryngeal and hypopharyngeal cancers are the most common malignant tumors of the head and neck in the world $[16,17]$. It was found that the fiveyear survival rate of laryngeal cancer patients has a close relationship with diagnostic staging. The survival rate and life quality of these patients can be improved by early diagnosis and treatment. Due to the absence of clinical tumor markers for laryngeal and hypopharyngeal cancers, the search for tumor markers has been a key factor influencing these patients, in terms of early diagnosis, early treatment and prognosis.

A growing number of studies have revealed that exosomes play a very important role in the immune function of cells, the intercellular transportation and the malignancy of cells $[18,19]$. Centrifugation, Exosome Isolation Kit and some similar kits have been the usual approaches performed to extract exosomes. The exosomes were successfully extracted from the peripheral blood serum of patients with laryngeal or hypopharyngeal cancer. The characteristics of a cup structure and size of $30-100 \mathrm{~nm}$ could be observed by transmission electron microscopy, which are in line with the features of exosomes. In the study conducted by Huang et al, they isolated and characterized the exosomes derived from the laryngeal squamous cell carcinoma cell line [20]. Our study also illustrated that tumor cells from laryngeal or hypopharyngeal cancer could secrete exosomes.

Cancer cells secrete at least 10 folds more exosomes than normal cells [21]. The components of tumor-derived exosomes are distinct from those released from normal cells [21]. And the size of exosomes from cancer cells seems larger than those from normal cells. In a previous study, elevated exosome sizes were detected in the saliva of patients with oral cancer [14]. As expected, in this study, we found that, compared with control group, both the volume and the particle size of exosomes were significantly increased in the laryngeal and hypopharyngeal cancers. Therefore, the size of peripheral blood exosome particles can be used as one of important indicators for the early diagnosis of laryngeal and hypopharyngeal cancers.

Exosome contains abundant proteins. In addition to self-proteins, it also contains proteins derived from cells $[22,23]$. Exosome proteins likely reflect their cellular origin and may also aid in the detection of cancer [21]. These proteins included constitutive components of exosomes such as tetraspanins (CD9, CD63, CD81, CD82, CD151, Tspan8), cell type-specific molecules such as histocompatibility complex (MHC) class-I and class-II and other molecules [11]. In the present study, it was found that the exosome protein concentration in the laryngeal and hypopharyngeal cancer groups was significantly higher than that in the control group. Furthermore, the exosome protein concentration of peripheral blood can be used as an indicator of laryngeal and hypopharyngeal cancers in the early diagnosis phase. When the protein concentration of exosomes is $\geqslant 2.8842 \mathrm{mg} / \mathrm{ml}$, the possibility of laryngeal or hypopharyngeal cancer should be taken into consideration.

Four-time transmembrane protein is one of the ideal protein markers of exosomes, which is highly expressed in exosomes. Both CD63 and CD81 are members of the four-time transmembrane protein family. Protein CD81 is a membrane surface protein involved in the expression of various biological functions of cells, such as cell membrane fusion and cell migration, adhesion, invasion, and proliferation [24]. The value of protein CD81 in tumor cells and hepatitis $C$ virus had been widely researched. Meanwhile, it has been found and verified that protein $\mathrm{CD} 81$ has a close relationship with the growth and invasion of tumors, and the infection of hepatitis $\mathrm{C}$ virus. Furthermore, recent studies have shown that exosomes expressing protein CD81 could regulate the Wnt-PCP signaling pathway, which is for the migration of breast cancer cells [25]. These show that protein CD81 plays an important role in the process of tumor cell migration. Furthermore, it was found that protein CD81 is also involved in the migration and invasion of skin keratinocytes [26]. However, few studies have been done on the influence of protein CD81 on the migration and invasion function of laryngeal cancer cells, especially on the migration and invasion function of exosomes. Protein CD63 is known as lysosomalassociated membrane protein 3 . It was found that protein CD63 is closely correlated to the invasion and metastasis of various tumors [27]. Previous studies have shown that the expression of protein CD63 is positively correlated with the differentiation degree of breast cancer, ovarian cancer, gastric cancer, and other cancers; and is negatively correlated with tumor invasion and metastasis [16]. Exosomeloaded protein CD63, which originates from tumors, might be a potential tumor marker. The present study focused on the high expression of proteins CD81 and CD63 in serum exosomes obtained from patients with laryngeal or hypopharyngeal cancer; 
and the results suggested that they are possible protein markers of these two types of cancer, which might play an important role in the processes of existence, growth and migration of malignant laryngeal tumor cells.

Limitations: Firstly, the present trial was not a randomized controlled trial. Secondly, the present study was merely a single-center trial, and the sample size was limited. Thirdly, all of the included patients were male. The value of exosomes in laryngeal or hypopharyngeal cancer among female patients remains unknown, and needs further research.

\section{CONCLUSION}

It was found that exosomes were expected to be a potential non-invasive and simple tumor marker of body fluid for laryngeal or hypopharyngeal cancer. This provides a direction for clinically accurate medical diagnosis.

Acknowledgements: The datasets and analyzed during the current study are available from the corresponding author on reasonable request. We thank the participants of the study. Funding: Zhejiang public technology application project (No. LGF19H130001); Zhejiang medical science and technology program (No. 2019KY743).

\section{REFERENCES}

1. Barbalata C, Mattos LS (2016) Laryngeal tumor detection and classification in endoscopic video. IEEE $J$ Biomed Health Inform 20, 322-332.

2. Toan NL, Hang NT, Luu NK, VAN Mao C, VAN Ba N, Xuan NT, Cam TD, Yamamoto N, et al (2019) Combination of vaccine strain measles virus and nimotuzumab in the treatment of laryngeal cancer. Anticancer Res 39, 3727-3737.

3. Pracy P, Loughran S, Good J, Parmar S, Goranova R (2016) Hypopharyngeal cancer: United Kingdom national multidisciplinary guidelines. $J$ Laryngol Otol 130, S104-S110.

4. Eckel HE, Bradley PJ (2019) Treatment options for hypopharyngeal cancer. Adv Otorhinolaryngol 83, 47-53.

5. Hong HJ, Jeong SH, Kim WS, Kim YJ (2019) Safety of esophagogastroduodenoscopy-guided forceps biopsy and the feasibility of esophagogastroduodenoscopy for evaluation of hypopharyngeal cancer. BMC Surg 19, ID 105.

6. Chen J, Hou H, Chen H, Luo Y, Zhang L, Zhang Y, Liu H, Zhang F, et al (2019) Urinary metabolomics for discovering metabolic biomarkers of laryngeal cancer using UPLC-QTOF/MS. J Pharm Biomed Anal $167,83-89$.

7. Topuz MF, Binnetoglu A, Yumusakhuylu AC, Sarı M, Baglam T, Gerin F (2017) Circulating calprotectin as a biomarker of laryngeal carcinoma. Eur Arch Otorhinolaryngol 274, 2499-2504.

8. Kalluri R (2016) The biology and function of exosomes in cancer. J Clin Invest 126, 1208-1215.

9. Tai YL, Chen KC, Hsieh JT, Shen TL (2018) Exosomes in cancer development and clinical applications. Cancer Sci 109, 2364-2374.

10. Bastos N, Ruivo CF, da Silva S, Melo SA (2018) Exosomes in cancer: use them or target them? Semin Cell Dev Biol 78, 13-21.

11. Xiao C, Song F, Zheng YL, Lv J, Wang QF, Xu N (2019) Exosomes in head and neck squamous cell carcinoma. Front Oncol 9, ID 894.

12. Koritzinsky EH, Street JM, Star RA, Yuen PS (2017) Quantification of exosomes. J Cell Physiol 232, 1587-1590.

13. Barile L, Vassalli G (2017) Exosomes: Therapy delivery tools and biomarkers of diseases. Pharmacol Ther 174, 63-78.

14. Sharma S, Gillespie BM, Palanisamy V, Gimzewski JK (2011) Quantitative nanostructural and singlemolecule force spectroscopy biomolecular analysis of human-saliva-derived exosomes. Langmuir 27, 14394-14400.

15. Kannan A, Hertweck KL, Philley JV, Wells RB, Dasgupta S (2017) Genetic mutation and exosome signature of human papilloma virus associated oropharyngeal cancer. Sci Rep 7, ID 46102.

16. Lupia A, Peppicelli S, Witort E, Bianchini F, Carloni V, Pimpinelli N, Urso C, Borgognoni L, et al(2014) CD63 tetraspanin is a negative driver of epithelial-tomesenchymal transition in human melanoma cells. $J$ Invest Dermatol 134, 2947-2956.

17. Tominaga N, Hagiwara K, Kosaka N, Honma K, Nakagama H, Ochiya T (2014) RPN2-mediated glycosylation of tetraspanin CD63 regulates breast cancer cell malignancy. Mol Cancer 13,ID 134.

18. Huang Y, Liu K, Li Q, Yao Y, Wang Y (2018) Exosomes function in tumor immune microenvironment. In: Mettinger K, Rameshwar P, Kumar V (eds) Exosomes, Stem Cells and MicroRNA. Advances in Experimental Medicine and Biology, vol 1056, Springer, Cham, pp 100-122.

19. Whiteside TL (2016) Exosomes and tumor-mediated immune suppression. J Clin Invest 126, 1216-1223.

20. Huang Q, Yang J, Zheng J, Hsueh C, Guo Y, Zhou L (2018) Characterization of selective exosomal microRNA expression profile derived from laryngeal squamous cell carcinoma detected by next generation sequencing. Oncol Rep 40, 2584-2594.

21. Li W, Li C, Zhou T, Liu X, Liu X, Li X, Chen D (2017) Role of exosomal proteins in cancer diagnosis. $\mathrm{Mol}$ Cancer 16, ID 145.

22. Bang C, Thum $T$ (2012) Exosomes: New players in cell-cell communication. Int J Biochem Cell Biol 44, 2060-2064. 
23. Raimondo F, Morosi L, Chinello C, Magni F, Pitto $M$ (2011) Advances in membranous vesicle and exosome proteomics improving biological understanding and biomarker discovery. Proteomics 11, 709-720.

24. Vences-Catalán F, Duault C, Kuo CC, Rajapaksa R, Levy R, Levy S (2017) CD81 as a tumor target. Biochem Soc Trans 45, 531-535.

25. Luga V, Zhang L, Viloria-Petit AM, Ogunjimi AA, Inanlou MR, Chiu E, Buchanan M, Hosein AN, et al (2012)
Exosomes mediate stromal mobilization of autocrine wnt-pcp signaling in breast cancer cell migration. Cell 151, 1542-1556.

26. Mazzocca A, Birgani MT, Sabbá C, Catloni V (2014) Tetraspanin-enriched microdomains and hepatocellular cancer progression. Cancer Lett 351, 23-29.

27. Sun Z, Wang L, Dong L, Wang X (2018) Emerging role of exosome signalling in maintaining cancer stem cell dynamic equilibrium. $J$ Cell Mol Med 22, 3719-3728. 